\title{
Uncertainty propagation and truncation errors in LPT kinematics
}

\author{
L. Chatellier ${ }^{1^{*}}$ \\ ${ }^{1}$ Institut PPRIME, UPR3346, CNRS - Université de Poitiers - ISAE-ENSMA, France \\ *ludovic.chatellier@univ-poitiers.fr
}

\begin{abstract}
Lagrangian Particle Tracking (LPT) has become a near-standard approach for performing accurate 3D flow measurements, thanks notably to the technical breakthroughs brought by the Iterative Particle Reconstruction (IPR: Wieneke, 2013) and Shake-the-Box (STB: Schanz et.al, 2016) procedures. These decisive progresses have triggered a number of studies relative to the eduction of flow kinematics and dynamics based on particle trajectory analyses. Novara \& Scarano (2013), and others, focused on polynomial approximations of the trajectories, which analytically provide the material derivatives used to estimate pressure gradients. In particular, approximations based on second order polynomials fits of a small number of particle positions are used in commercially available softwares and among research teams as a straightforward solution to obtain the first and second order derivatives with a limited effect of the measurement noise. Additionally the analyses conducted during the 2020 LPT challenge (Leclaire, 2020 ; Sciacchitano, 2020) have addressed the performance of methodologies used by different groups with respect to second order trajectory fits for both multi-pulse and four-pulse (Novara et. al, 2016) LPT cases. On more advanced theoretical grounds, Geseman et. al (2016) have proposed the trackfit approach using penalized B-splines with considerations on the time-varying acceleration rate (i.e. jolt or jerk) and spectral content of noisy particle tracks.

From a general point of view on function approximations, polynomial fit, Taylor developments and finite differences schemes are absolutely equivalent ingredients of a unique framework in which discrete sets of ordered values are represented by continuous functions of a single parameter. This can be easily recalled using linear algebra applied to the construction of finite difference schemes and to the evaluation of their orders of truncation as well as their sensitivity to measurement noise. The present contribution provides a practical methodology that can be included in a global uncertainty quantification framework. Examples illustrating a variety of schemes are presented, along with suggestions of analytical tests based on theoretical results.
\end{abstract}

\section{Introduction}

Within the Lagrangian Particle Tracking (LPT) framework, the time-history spatial positions of the individually identified particles are used to constitute discrete tracks and compute their trajectories as well as their kinematics. In pratice, acquisition sequences consist in two-, four- or multi-pulse exposures (resp. TP, FP and MP) resulting in two, four or multiple successive measured positions of a single particle in space, as depicted in Figure 1. 


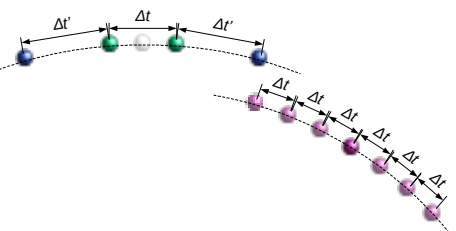

Figure 1: Typical distribution of two- (green) four- (blue) and multi-pulse (purple) LPT measurement time lags.

In conventional PTV algorithms, TP measurements are used to derive central positions and velocities using second-order differentiation schemes, thanks to the symmetry properties of the 2point centered finite differences formulation. A minimum of three positions is needed to obtain acceleration using second order derivation schemes. For FP and MP (i.e more than two pulses) LPT measurements, higher order derivatives are accessible using second or higher order derivations schemes. Apart from the jolt which is sometimes studied, the quantities of interest are most often limited to position, velocity and acceleration. It is also of common practice to model trajectories as piece-wise polynomials of degree ranging from 2 to a few units, from which these quantities are derived. While finite differences derivation schemes at various orders are long known, they are often presented as well-chosen linear combinations of Taylor developments from which a particular derivative is obtained. Elements of the Richardson expansion are also sometimes used to build the linear combinations that can increase the truncation order of a given scheme. The formalism used below aims at rationalizing the practical writing of differentiation schemes with a view to ease the estimation of truncation errors, the propagation of measurement noise and uncertainty, and a direct correspondence with polynomial approximations.

\section{Taylor development and polynomial fit}

The estimation of derivatives using polynomial approximations or schemes based on Taylor developments are perfectly equivalent approaches. This is recalled below in the context of discretized trajectories.

Given a set of particle positions $x_{k}$ belonging to the same trajectory $\Gamma$ as obtained from TRPTV or LPT, positions, velocities, accelerations and other derivatives can be educed from linear combinations of the measured positions using either finite differences or polynomial fits.

Given an instant of interest $t$ and a distribution of time lags $\tau_{k}$ at which particle positions are measured, one writes :

$$
\forall \tau_{k}, x_{k}=\Gamma\left(t+\tau_{k}\right)+e_{k}
$$

where $e_{k}$ is the measurement error on the particle position.

Within a set $T_{m}=\left\{t+\tau_{1} ; \ldots ; t+\tau_{m}\right\}$ of $m$ measurement instants, the corresponding measured and true positions are respectively written as $X_{m}=\left[\begin{array}{lll}x_{1} & \ldots & x_{m}\end{array}\right]^{\top}$ and $G_{m}=\left[\begin{array}{llll}\Gamma\left(t+\tau_{1}\right) & \ldots & \Gamma\left(t+\tau_{m}\right)\end{array}\right]^{\top}$ so that $X_{m}=G_{m}+E_{m}$.

The Taylor development of order $n \in \mathbb{N}^{+}$of the true positions around instant $t$ then writes: 


$$
\forall n \in \mathbb{N}^{+}, \forall \tau_{k}, \Gamma\left(t_{0}+\tau_{k}\right)=\Gamma(t)+\tau_{k} \dot{\Gamma}(t)+\ldots+\tau_{k}^{n} \Gamma^{(n)}(t) / n !+o\left(\tau_{k}^{n}\right)
$$

Yielding, for the set $T_{m}$ :

$$
G_{m}=\left[\begin{array}{cccc}
1 & \tau_{1} & \ldots & \tau_{1}^{n} \\
\vdots & & & \vdots \\
1 & \tau_{m} & \ldots & \tau_{m}^{n}
\end{array}\right]\left[\begin{array}{c}
\Gamma(t) \\
\vdots \\
\Gamma^{(n)}(t) / n !
\end{array}\right]+o\left(\begin{array}{c}
\tau_{1}^{n} \\
\vdots \\
\tau_{m}^{n}
\end{array}\right)
$$

Similarly, a polynomial $P$ of degree $n$ fitting positions $G_{m}$ according to a given norm can be defined from its coefficients $\left(a_{k}\right)_{k=0 \ldots n}$ and written for the set $T_{m}$ as:

$$
\left[P\left(\tau_{p}\right)\right]_{p=1 \ldots m}=\left[\begin{array}{ccccc}
1 & \ldots & \tau_{1}^{k} & \ldots & \tau_{1}^{n} \\
\vdots & & & & \vdots \\
1 & \ldots & \tau_{m}^{k} & \ldots & \tau_{m}^{n}
\end{array}\right]\left[\begin{array}{c}
a_{0} \\
\vdots \\
a_{n}
\end{array}\right]
$$

Identifying coefficients $\left(a_{k}\right)_{k=0 \ldots n}$ to the successive derivatives of $P$ gives:

$$
\forall k \in\{0 \ldots n\}, \quad a_{k}=\frac{P^{(k)}(0)}{k !} .
$$

So that

$$
\left[P\left(\tau_{p}\right)\right]_{p=1 \ldots m}=\left[\begin{array}{ccccc}
1 & \ldots & \tau_{1}^{k} & \ldots & \tau_{1}^{n} \\
\vdots & & & & \vdots \\
1 & \ldots & \tau_{m}^{k} & \ldots & \tau_{m}^{n}
\end{array}\right]\left[\begin{array}{c}
P(0) \\
\dot{P}(0) \\
\vdots \\
P^{(n)}(0) / n !
\end{array}\right]
$$

For the positions $G_{m}$ and derivatives $\left(\left.\Gamma^{(k)}(t)\right|_{k=0 \ldots n}\right.$ to be approximated by polynomial $P$, one therefore gets the same matrix formulation as the one provided by the Taylor development of $G_{m}$ around instant $t$. The formal difference between the two approaches lies in the presence of truncation terms in the Taylor development and, implicitly, of a possible fitting error in the polynomial approximation.

\section{System inversion}

The linear relation between the positions and derivatives is fully contained in the full or truncated Vandermonde matrix constructed from the time lags. For an equal number of positions and derivatives terms $(m=n+1)$, the matrix is complete and invertible provided that all time lags are distinct from each other. The polynomial therefore provides an exact fit of the measured positions. For reduced polynomial degrees or orders of truncation (i.e. $m>n+1$ ), the truncated Vandermonde matrix is still left-invertible so that the generalized or the Moore-Penrose pseudoinverse can be used to identify the successive derivatives.

In the following paragraphs, a reference time lag $d t$ will be used in order to represent each time lag $\tau_{k}$ by $\tau_{k}=\alpha_{k} d t$ with $\forall k \in \mathbb{N}, \alpha_{k} \in \mathbb{N}$, as represented in Figure 2 . 


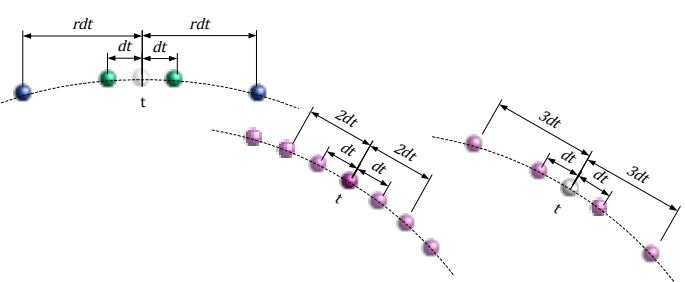

Figure 2: Definition of two- (green) four- (blue) and odd or even multi-pulse (purple) LPT measurement time lags referenced to a central measurement time, as used in finite difference schemes.

The Taylor development now writes:

$$
G_{m}=\left[\begin{array}{cccc}
1 & \alpha_{1} & \ldots & \alpha_{1}^{n} \\
\vdots & & & \vdots \\
1 & \alpha_{m} & \ldots & \alpha_{m}^{n}
\end{array}\right]\left[\begin{array}{c}
\Gamma(t) \\
\vdots \\
\Gamma^{(n)}(t) d t^{n} / n !
\end{array}\right]+o\left(d t^{n}\right)
$$

For a square system, the $m \times m$ Vandermonde matrix $V\left(\alpha_{1}, \ldots, \alpha_{m}\right)$ will be denoted $V_{m}$ and its inverse $W_{m}$. For $m>n+1$, the $m \times(n+1)$ truncated Vandermonde matrix will be denoted $V_{m n}$ and its $(n+1) \times m$ pseudo-inverse $W_{n m}$, noticing that $V_{m}=V_{m m-1}$ and $W_{m}=W_{m-1 m}$. For the estimation of truncation errors, $m \times p(p>n)$ matrices $V_{m p}$ will be used, which are either right-truncated, square or right-extended Vandermonde matrices, depending on the values of $m$, $n$ and $p$.

Defining $A_{m}=\left[\begin{array}{lll}\alpha_{1} & \ldots & \alpha_{m}\end{array}\right]^{\top}$ and $D_{n}=\left[\begin{array}{llll}\Gamma(t) & \ldots & \Gamma^{(n)}(t) d t^{n} / n !\end{array}\right]^{\top}$, the Taylor development can also be formulated as: $G_{m}=V_{m n} D_{n}+o\left(d t^{n}\right)=\left[\begin{array}{lll}A_{m}^{0} & \ldots & A_{m}^{n}\end{array}\right] D_{n}+o\left(d t^{n}\right)$.

\subsection{Exact fit}

For $m=n+1$, the Taylor development can be inverted to provide $V_{m}^{-1} G_{m}=D_{m}+o\left(d t^{n}\right)$, so that

$$
D_{m}=W_{m} G_{m}+o\left(d t^{n}\right)
$$

The differentiation schemes at all orders are therefore contained in the weighting matrix $W_{m}$, modulated by the temporal and factorial terms, to provide an estimate of the derivatives as:

$$
\left[\begin{array}{llll}
\widetilde{\Gamma}(t) & \ldots & \widetilde{\Gamma}^{(n)}(t)
\end{array}\right]^{\top}=\operatorname{diag}\left(\left(k ! / d t^{k}\right)_{k=0 \ldots m-1}\right) W_{m} X_{m}=F_{m} W_{m} X_{m} .
$$

Formally, the order of truncation is equal to $n=m-1$ but can drop to lower values depending on the distribution of the time lags. 


\subsubsection{Error propagation:}

In the specific case of an exact fit, the Taylor expansion at order $n=m-1$ and the polynomial fit of the same degree fall exactly on the measured positions $X_{m}$, inducing no approximation error. It is then straightforward to educe the levels of error propagation due the differentiation schemes as:

$$
u_{m}=F_{m} W_{m} E_{m} .
$$

This formulation evidences the increasing sensitivity to measurement errors with increasing truncation orders, as a by-product of the well-known Runge phenomenon occurring in increasing order polynomial interpolations

\subsubsection{Truncation error:}

The truncation error at order $p>n+1$ can be estimated using supplementary Taylor terms :

$$
G_{m}=\left[\begin{array}{llll}
A_{m}^{0} & A_{m}^{1} & \ldots & A_{m}^{p}
\end{array}\right]\left[\begin{array}{c}
\Gamma(t) \\
\vdots \\
\Gamma^{(p)}(t) d t^{p} / p !
\end{array}\right]+o\left(d t^{m}\right)=V_{m p} D_{p}+o\left(d t^{p}\right)=\left[\begin{array}{llll}
V_{m} & A_{m}^{n+1} & \ldots & A_{m}^{p}
\end{array}\right] D_{p}+o\left(d t^{p}\right)
$$

Inverting the system one obtains:

$$
W_{m} G_{m}=W_{m}\left[\begin{array}{llll}
V_{m} & A_{m}^{n+1} & \ldots & A_{m}^{p}
\end{array}\right] D_{p}+o\left(d t^{p}\right)=W_{m}\left[\begin{array}{llll}
V_{m} & A_{m}^{m+1} & \ldots & A_{m}^{p}
\end{array}\right]\left[\begin{array}{c}
D_{n+1} \\
\vdots \\
\Gamma^{(p)} d t^{p} / p !
\end{array}\right]+o\left(d t^{p}\right)
$$

and

$$
D_{n+1}=W_{m} G_{m}-W_{m}\left[\begin{array}{lll}
A_{m}^{n+1} & \ldots & A_{m}^{p}
\end{array}\right]\left[\Gamma^{(n+1)} d t^{n+1} /(n+1) ! \ldots \Gamma^{(p)} d t^{p} / p !\right]^{\top}+o\left(d t^{p}\right)
$$

Hence, truncation errors at order $m=n+1$ and above are given by :

$$
\begin{gathered}
\forall p>n+1, \\
F_{m} W_{m}\left[A_{m}^{n+1} \ldots A_{m}^{p}\right]\left[\Gamma^{(n+1)} d t^{n+1} /(n+1) ! \ldots \Gamma^{(p)} d t^{p} / p !\right]^{\top}+o\left(d t^{p}\right)=F_{m} W_{m} A_{m}^{n+1 \rightarrow p} D_{n+1 \rightarrow p}+o\left(d t^{p}\right)
\end{gathered}
$$

Considering the derivation terms (including the $0^{\text {th }}$ order term), the remaining truncation error terms can be different for each derivation order depending on the distribution of time lags.

\subsection{Approximate fit}

Extending this approach to lower order estimates in which $n<m-1$ is almost identical, although the Vandermonde matrix will not be fully obtained and an over-constrained system will be defined. However, as right-truncated Vandermonde matrices are always left-invertible, their pseudo-inverse writes:

$$
W_{n m}=\left(V_{m n}^{\top} V_{m n}\right)^{-1} V_{m n}^{\top} \text { so that } W_{n m} V_{m n}=1_{n+1}
$$


The pseudo-inverse $W_{n m}$ provides the differentiation scheme to be used to fit the trajectory and its $n$ first derivatives, as $G_{m}=V_{m n} D_{n}+o\left(d t^{n}\right)$ and $W_{n m} G_{m}=D_{n}+o\left(d t^{n}\right)$. The derivatives are estimated using

$$
\left[\begin{array}{llll}
\widetilde{\Gamma}(t) & \ldots & \widetilde{\Gamma}^{(n)}(t)
\end{array}\right]^{\top}=F_{n+1} W_{n m} X_{m}
$$

Hence, the levels of error propagation due the differentiation schemes are: $u_{n+1}=F_{n+1} W_{n m} E_{m}$.

The truncation error at order $p>n+1$ are given by:

$$
\forall p>n+1, \quad F_{n+1} W_{n m} A_{m}^{n+1 \rightarrow p} D_{n+1 \rightarrow p}+o\left(d t^{p}\right) .
$$

The approximate fit therefore directly generalizes the exact fit using either the standard- or pseudo-inverses of Vandermonde matrices constructed from a Taylor development.

\subsection{Examples}

The application of these procedures are easily illustrated using examples on common use. For TP measurements, the two-point centered derivation scheme leads to $3^{\text {rd }}$ order truncation errors for the first derivative, although it is based on a first order development. For MP measurements, the three-point centered derivation schemes leads to the same stencil for the first derivative, although it is based on a second order development. Acknowledging the fact that estimates are generally conducted on larger, centered, regular sets of instants in MP measurements, the three-point case is sufficient to identify which terms are to be taken into account for uncertainty and error estimates. A dedicated example is also given for the specific case of FP measurements, using a generic centered distribution of instants, and which illustrates the slight differences between exact and approximate fits of close orders.

\subsubsection{Exact fit}

\section{Two-point centered scheme (TP):}

For the two-point centered scheme built from instants $t-d t$ and $t+d t$, the differentiation scheme is simply obtained from the Taylor terms at order 1:

$$
G_{2}=\left[\begin{array}{rr}
1 & -1 \\
1 & 1
\end{array}\right]\left[\begin{array}{c}
\Gamma(t) \\
\dot{\Gamma}(t) d t
\end{array}\right]+o(d t) ; \quad V_{2}=\left[\begin{array}{cc}
1 & -1 \\
1 & 1
\end{array}\right] ; \quad W_{2}=\left[\begin{array}{cc}
1 / 2 & 1 / 2 \\
-1 / 2 & 1 / 2
\end{array}\right]
$$

For this particular case, and more generally for centered distributions of an even numbers of instants, halving $d t$ lightens the writing of the time lags surrounding the instant of interest (see Figure 2).

The central position and first derivative are estimated using $[\widetilde{\Gamma}(t) \tilde{\dot{\Gamma}}(t)]^{\top}=F_{2} W_{2} X_{2}$, and the propagation of measurement errors is given by $u_{2}=F_{2} W_{2} E_{2}$ :

$$
u_{2}=\left[\begin{array}{cc}
1 & 0 \\
0 & 1 / d t
\end{array}\right]\left[\begin{array}{cc}
1 / 2 & 1 / 2 \\
-1 / 2 & 1 / 2
\end{array}\right] E_{2}=\left[\begin{array}{cc}
1 / 2 & 1 / 2 \\
-1 / 2 d t & 1 / 2 d t
\end{array}\right] E_{2}
$$


For uniform error levels, this yields error propagation terms of respective norms $\left[\begin{array}{ll}1 & 1 / d t\end{array}\right]^{\top} / \sqrt{2}$.

The truncation error on both terms is obtained by right-extending the $2 \times 2$ Vandermonde matrix to the $2 \times 4$ matrix $V_{23}$ and multiplying it by the finite difference scheme:

$$
W_{2} V_{23}=\left[\begin{array}{cc}
1 / 2 & 1 / 2 \\
-1 / 2 & 1 / 2
\end{array}\right]\left[\begin{array}{cccc}
1 & -1 & 1 & -1 \\
1 & 1 & 1 & 1
\end{array}\right]=\left[\begin{array}{llll}
1 & 0 & 1 & 0 \\
0 & 1 & 0 & 1
\end{array}\right] \text {, }
$$

meaning that the central position is obtained with a $2^{\text {nd }}$ order truncation error and the first derivative with a $3^{\text {rd }}$ order truncation error. Applied to the differentiation terms up to the $3^{\text {rd }}$ order, this explicitly yields:

$$
F_{2} W_{2} V_{23} D_{3}=\left[\begin{array}{ccc}
\Gamma(t) & + & \ddot{\Gamma}(t) d t^{2} / 2 \\
\dot{\Gamma}(t) & + & \Gamma^{(3)}(t) d t^{2} / 6
\end{array}\right]+o\left(d t^{2}\right)
$$

\section{Three-point centered scheme (MP):}

For the three-point centered scheme built from instants $t, t-d t$ and $t+d t$ :

$$
G_{3}=\left[\begin{array}{rrr}
1 & -1 & 1 \\
1 & 0 & 0 \\
1 & 1 & 1
\end{array}\right]\left[\begin{array}{c}
\Gamma(t) \\
\dot{\Gamma}(t) d t \\
\ddot{\Gamma}(t) d t^{2} / 2
\end{array}\right]+o(d t) \quad ; \quad V_{2}=\left[\begin{array}{rrr}
1 & -1 & 1 \\
1 & 0 & 0 \\
1 & 1 & 1
\end{array}\right] ; \quad W_{3}=\left[\begin{array}{ccc}
0 & 1 & 0 \\
-1 / 2 & 0 & 1 / 2 \\
1 / 2 & -1 & 1 / 2
\end{array}\right] .
$$

The propagation of measurement errors is given by $u_{3}=F_{3} W_{3} E_{3}$ :

$$
u_{3}=\left[\begin{array}{ccc}
0 & 1 & 0 \\
-1 / 2 d t & 0 & 1 / 2 d t \\
1 / d t^{2} & -2 / d t^{2} & 1 / d t^{2}
\end{array}\right] E_{3}
$$

of norms $\left[1 \frac{\sqrt{2}}{2 d t} \frac{\sqrt{6}}{d t^{2}}\right]^{\top}$ for uniform noise levels.

The truncation error at order 4 is obtained from :

$$
W_{3} V_{34}=\left[\begin{array}{lllll}
1 & 0 & 0 & 0 & 0 \\
0 & 1 & 0 & 1 & 0 \\
0 & 0 & 1 & 0 & 1
\end{array}\right]
$$

The central position is obtained with no truncation error, as it is measured prior to building the differentiation scheme. This property also distinguishes centered schemes based on odd or even numbers of instants. The first derivative is obtained with a $3^{\text {rd }}$ order truncation error, and the second derivative with a $4^{\text {th }}$ order truncation error. One gets: 


$$
F_{3} W_{3} V_{34} D_{3}=\left[\begin{array}{l}
\Gamma(t) \\
\dot{\Gamma}(t)+\Gamma^{(3)}(t) d t^{2} / 6+o\left(d t^{3}\right) \\
\ddot{\Gamma}(t)+\Gamma^{(4)}(t) d t^{2} / 12+o\left(d t^{2}\right)
\end{array}\right]
$$

It can be noticed that the value of $d t$ is doubled compared to the two-point scheme obtained with the same sampling, multiplying the error levels and truncation errors by factors $2^{ \pm 1 \ldots 2}$.

\section{Generic four-point centered scheme (FP):}

For centered FP measurements, time lags are symmetrically defined as $\left(\tau_{k} / d t\right)_{k=1 \ldots 4}=\{-a ;-b ; b ; a\}$ with $a>b>0$. One can set $b=1$ and change $d t$ accordingly without loss of generality to introduce the ratio $r=a / b \neq 1$ which is sufficient to describe any centered FP distribution. As for the TP case, halving $d t$ lightens the writing of the time lags surrounding the instant of interest.

The exact fit is obtained from a Taylor development at the $3^{\text {rd }}$ order in which:

$$
V_{4}=\left[\begin{array}{cccc}
1 & -r & r^{2} & -r^{3} \\
1 & -1 & 1 & -1 \\
1 & 1 & 1 & 1 \\
1 & r & r^{2} & r^{3}
\end{array}\right]
$$

Here, setting $r= \pm 3$ or $r= \pm 1 / 3$ transforms the generic FP case in a regularly distributed fourinstants MP case, which can be generalized to centered MP measurements based on an even number of instants.

This yields the inverse matrix:

$$
W_{4}=\frac{1}{2 r\left(r^{2}-1\right)}\left[\begin{array}{cccc}
-r & r^{3} & r^{3} & -r \\
1 & -r^{3} & r^{3} & -1 \\
r & -r & -r & r \\
-1 & r & -r & 1
\end{array}\right]
$$

and gives the propagation error term $u_{4}=F_{3} W_{4} E_{4}$ :

$$
\begin{aligned}
& u_{4}=\frac{1}{2 r\left(r^{2}-1\right)}\left[\begin{array}{cccc}
1 & 0 & 0 & 0 \\
0 & 1 / d t & 0 & 0 \\
0 & 0 & 2 / d t^{2} & 0 \\
0 & 0 & 0 & 6 / d t^{3}
\end{array}\right]\left[\begin{array}{cccc}
-r & r^{3} & r^{3} & -r \\
1 & -r^{3} & r^{3} & -1 \\
r & -r & -r & r \\
-1 & r & -r & 1
\end{array}\right] E_{4}, \\
& \text { of norms: } \frac{1}{\left|r^{2}-1\right|}\left[\frac{\sqrt{r^{4}+1}}{\sqrt{2}} \frac{\sqrt{r^{6}+1}}{\sqrt{2}|r| d t} \frac{2}{d t^{2}} \frac{3 \sqrt{2\left(r^{2}+1\right)}}{|r| d t^{3}}\right]^{\top} \text {. }
\end{aligned}
$$

The corresponding truncation errors at order 5 are given by: 


$$
F_{4} W_{4} V_{45} D_{5}=\left|\begin{array}{ccccc}
\Gamma(t) & - & r^{2} \frac{d t^{4}}{24} \Gamma^{(4)}(t) & +o\left(d t^{5}\right) \\
\dot{\Gamma}(t) & - & r^{2} \frac{d t^{4}}{120} \Gamma^{(5)}(t)+o\left(d t^{4}\right) \\
\ddot{\Gamma}(t) & + & \left(r^{2}+1\right) \frac{d t^{2}}{12} \Gamma^{(4)}(t)+o\left(d t^{2}\right) \\
\Gamma^{(3)}(t) & + & \left(r^{2}+1\right) \frac{d t^{2}}{20} \Gamma^{(5)}(t)+o\left(d t^{2}\right)
\end{array}\right|
$$

The central position and acceleration are obtained with a truncation error of $4^{\text {th }}$ order, respectively proportional to $r^{2}$ and $r^{2}+1$. Velocity and jolt are obtained with a truncation error of $5^{\text {th }}$ order, also respectively proportional to $r^{2}$ and $r^{2}+1$.

\subsubsection{Approximate fit}

For FP or MP measurements, the second order fit is mandatory but sufficient to obtain $2^{\text {nd }}$ order derivatives. It can be used to illustrate approximate fits at all orders below $m$.

Apart from the exact $m=n+1=3$ case illustrated above, it can be written from $V_{m 2}=\left[\begin{array}{lll}A_{m}^{0} & A_{m}^{1} & A_{m}^{2}\end{array}\right]$ whose pseudo-inverse is $W_{2 m}$. Error propagation is directly given by writing $F_{3} W_{2 m} E_{m}$ and truncation errors from:

$$
W_{2 m} G_{m}=D_{2}+W_{2 m} A_{m}^{3} \Gamma^{(3)} d t^{3} / 3 !+\ldots+W_{2 m} A_{m}^{(p)} \Gamma^{(p)} d t^{p} / p ! o\left(d t^{p}\right)
$$

For odd MP distributions, the construction and analysis of the central finite differences scheme is similar to what is obtained for the exact $m=n+1=3$ case.

For even MP distribution, the construction of the centered finite difference scheme can be illustrated by a second order approximate fit of the generic FP case. The corresponding Taylor development is built as a restriction of $V_{4}$ used in the exact FP case:

$$
V_{42}=\left[\begin{array}{ccc}
1 & -r & r^{2} \\
1 & -1 & 1 \\
1 & 1 & 1 \\
1 & r & r^{2}
\end{array}\right]
$$

which results in the pseudo-inverse matrix:

$$
W_{24}=\frac{1}{2}\left[\begin{array}{cccc}
-\frac{1}{r^{2}-1} & \frac{r^{2}}{r^{2}-1} & \frac{r^{2}}{r^{2}-1} & -\frac{1}{r^{2}-1} \\
-\frac{r}{r^{2}+1} & -\frac{1}{r^{2}+1} & \frac{1}{r^{2}+1} & \frac{r}{r^{2}+1} \\
\frac{1}{r^{2}-1} & -\frac{1}{r^{2}-1} & -\frac{1}{r^{2}-1} & \frac{1}{r^{2}-1}
\end{array}\right],
$$


and in which only the first derivative weights differ from $W_{4}$.

The error propagation term is $u_{4}=F_{3} W_{24} E_{4}$, of norms: $\left[\frac{\sqrt{r^{4}+1}}{\sqrt{2}\left|r^{2}-1\right|} \frac{1}{\sqrt{2\left(r^{2}+1\right)} d t} \frac{2}{\left|r^{2}-1\right| d t^{2}}\right]$.

The truncation error is given by:

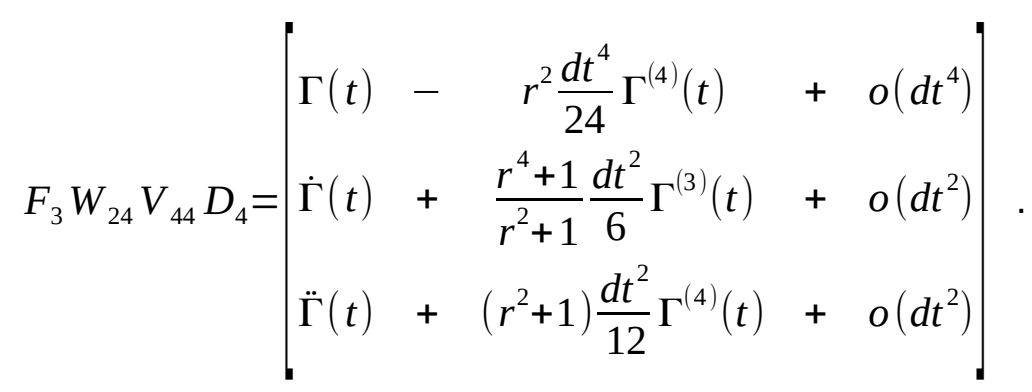

Compared to the exact FP scheme, the estimates of the position and acceleration are identical. The difference between the two schemes only appear in the velocity estimates, which are obtained at order 3 in the approximate fit and order 4 in the exact fit. Conversely, the error propagation on the velocity term is governed by:

$$
\frac{\sqrt{r^{6}+1}}{\sqrt{2}|r|\left|r^{2}-1\right| d t} \text { for the exact fit, }
$$

and

$$
\frac{1}{\sqrt{2\left(r^{2}+1\right) d t}} \text { for the approximate fit. }
$$

It is then clear that despite the lower truncation error, the $2^{\text {nd }}$ order fit results in a significantly lower sensitivity to measurement errors, of at least a factor of the order of 3 depending on the chosen value for $r$. This result is also a by-product of the Runge phenomenon.

For the choice of an optimal value of $r$ and an exact or approximate fit, some knowledge on the $3^{\text {rd }}$ derivative can be obtained from the exact fit and compared with the propagation of measurement noise, however anticipating the choice of $r$ before an experiment may not be straightforward unless numerical simulations or theoretical results can help estimate the balance between the two terms.

For higher order schemes on MP measurement leading to long tracks, the orders of derivation needed to estimate the truncation error are accessible by simply increasing the order of the scheme of interest by a few units. Thus, and accounting for the increased error propagation due to the Runge phenomenon, all the needed terms can be estimated using:

$$
u_{n+1}=F_{n+1} W_{n m} E_{m} \text { and } \forall p>n+1, F_{n} W_{n m} A_{m}^{n+1 \rightarrow p} D_{n+1 \rightarrow p}+o\left(d t^{p}\right) .
$$

In order to obtain higher order reference derivatives, trajectories may be approximated by alternative, non-polynomial functions. However, the large spectrum of possibilities doesn't help 
providing the most appropriate formulation. Analytical flow solutions, though limited to idealized or approximate configurations, may provide the relevant results for a given situation. A few examples are proposed below.

\subsubsection{Error estimates from analytical trajectories}

Finite difference schemes and error estimates as obtained from the above formulations may be benchmarked against known trajectories from which any order of differentiation can be expressed. Apart for specific cases in which vanishing derivatives are a constitutive property, such as, e.g, resting, uniform or unidirectional flow, free fall, etc., non-polynomial analytic trajectories can provide the derivatives needed to qualify a given differentiation scheme.

Basic non-polynomial examples can be found from potential flows, such as the isolated vortex or normal stagnation points which provide trajectories as steady state streamlines. Unsteady potential flows and the corresponding trajectories can also be obtained by imposing time-dependent parameters. Stream-functions can also provide integrable analytical solutions for a variety of 2D viscous flows, including boundary layers (Blasius, 1908) and non-orthogonal stagnation points (Dorrepal, 1986). However, in many formulations, explicit time-dependent trajectories cannot be found and must be numerically integrated, leading to additional error terms, though the successive derivatives remain accessible.

A universal illustration is provided by the isolated vortex for which trajectories are described by harmonic terms, with or without advection velocity, to provide circular or cycloid-like tracks. The truncation terms are therefore obtained from the harmonic terms around the measurement instant. For example, expanding $\forall \tau_{k}, \Gamma\left(t+\tau_{k}\right) \propto \sin \left(\omega\left(t+\tau_{k}\right)\right)$ and applying the desired finite difference scheme, then substracting the Taylor terms, will yield and exact expression of the truncated terms.

Similarly, the 2D inviscid flow normally impinging on a flat plate is represented by potential $\varphi \propto x^{2}-y^{2}$, stream-function $\psi \propto x y$ or potential $f(z) \propto z^{2}$ in complex formulation, leading to exponential streamlines that can be expanded around the measurement instant using hyperbolic functions. Despite its simplicity, this potential is representative of the flow in the close vicinity of stagnation or separation points on cylinders or wing profiles. For example, the 2D complex potential of the flow around a cylinder, yields near the stagnation point:

$$
f(z)=U_{0}\left(z+\frac{a^{2}}{z-a}\right): f(z) \underset{z \rightarrow 0}{\rightarrow}-U_{0} \frac{z^{2}}{a},
$$

locally leading to exponential trajectories. This can be extended to non-orthogonal impingement using a stream-function of type $\psi \propto \frac{1}{2} y^{2} \cos \alpha+x y \sin \alpha$ to provide combinations of exponential trajectories of type:

$$
x=x_{0} e^{\frac{t}{T} \sin \alpha}+\frac{1}{2} y_{0} \cot \alpha \operatorname{sh}\left(\frac{t}{T} \sin \alpha\right) ; y=y_{0} e^{-\frac{t}{T} \sin \alpha} .
$$

Finally, in the close vicinity of a flat plate, the Blasius boundary layer classically represented by the stream-function $\psi(x, y)=\delta(x) U f(y / \delta(x))$ leads to power-law trajectories:

$$
x=x_{0}\left(1+\frac{5}{4} U \sqrt{\operatorname{Re}_{0}} \frac{y_{0}}{x_{0}^{2}} \alpha \tau\right)^{4 / 5} ; y=y_{0}\left(1+\frac{5}{4} \frac{y_{0}}{x_{0}^{2}} U \sqrt{\operatorname{Re}_{0}} \alpha \tau\right)^{1 / 5}
$$


for $f(\eta)=\frac{1}{2} \alpha \eta^{2}+O\left(\eta^{5}\right)$ and $y \ll \delta(x)$. Since PTV or LPT shall be preferred to PIV measurements in order to resolve near wall velocity fields (Kaelher, 2012), this last formulation may be used to predict uncertainty on a planned measurement, or to fit and qualify existing results.

\section{Conclusion}

Although finite differences schemes are long known and widely used in the scientific communities for experimental and numerical studies, little attention is generally paid to their content in terms of error propagation and actual truncation terms, and to their relevance to data fitting. The present contribution provides a straightforward and practical mean to access and qualify these aspects using linear algebra, with the possibility of analytical quantification. Additionally, the resulting formulations can be easily extended to 2D or 3D for post-processing dense, regular, spatial fields. In this perspective, the observations of, e.g., Raffel et. al (1998) on the sensitivity of derivation results obtained from schemes based either on Richardson expansion or least-square fits are formally explained by the balance between error propagation and truncation terms.

\section{Acknowledgements}

This project has received funding from the European Research Council (ERC) under the European Union's Horizon 2020 research and innovation programme, project $H \diamond M<R$ : Holistic Optical Metrology for Aero-Elastic Research (grant agreement No 648161).

\section{References}

Acher G, Thomas L, Tremblais B, Gomit G, Chatellier L, David L, Simultaneous measurements of flow velocity using Tomo-PIV and deformation of a flexible wing, 13th International Symposium on Particle Image Velocimetry, 2019

Blasius $\mathrm{H}$, The Boundary Layer in fluids with little friction (Translation of Grenzschichten in Fliissigkeiten mit kleiner Reibung, Zeitschrift fixr Mathematik und Physik, Band 56, Heft 1, 1908), NACA TM-1256, 1950

Raffel M, Willert M, Kompenhans J., Particle Image Velocimetry, A Practical Guide, Springer-Verlag Berlin, Heidelberg, 1998

Dorrepal J M, An exact solution of the Navier-Stokes equation which describes non-orthogonal stagnation-point flow in two dimensions, J. Fluid Mech. 163:141-147, 1986

Gesemann S, Huhn F, Schanz D, Schröder A, From Noisy Particle Tracks to Velocity, Acceleration and Pressure Fields using B-splines and Penalties, 18th International Symposium on the Application of Laser and Imaging Techniques to Fluid Mechanics, 2016

Kähler C J, Scharnowski S, Cierpka C, On the uncertainty of digital PIV and PTV near walls, Experiments in Fluids 52:1641-1656, 2012

Leclaire $B$, Challenge datasets generation: physical situation, numerical simulation and synthetic generation, $3^{\text {rd }}$ Workshop and $1^{\text {st }}$ Challenge on Data Assimilation \& CFD Processing for PIV and Lagrangian Particle Tracking, 2020

Neagoe VE, Inversion of the Van der Monde Matrix, IEEE Signal Processing Letters 3:4, 1996

Novara M, Scarano F, A particle-tracking approach for accurate material derivative measurements with tomographic PIV. Exp.Fluids 54:158, 2013

Novara M, Schanz D, Reuther N, Kähler CJ, Schröder A, Lagrangian 3D particle tracking in high-speed flows: Shake-The-Box for multi-pulse systems, Experiments in Fluids 57(8), 2016

Schanz D, Gesemann S, Schröder A, Shake-The-Box: Accurate Lagrangian particle tracking at high particle densities. Exp.Fluids 57:70, 2016 
14th International Symposium on Particle Image Velocimetry - ISPIV 2021

August 1-5, 2021

Sciacchitano A, LPT Challenge results, $3^{\text {rd }}$ Workshop and $1^{\text {st }}$ Challenge on Data Assimilation \& CFD Processing for PIV and Lagrangian Particle Tracking, 2020

Wieneke B, Iterative reconstruction of volumetric particle distribution. Meas. Science Tech. 24,024008, 2013 